\title{
Defining linkage to care following human immunodeficiency virus (HIV) diagnosis for public health monitoring in Europe
}

Sara Croxford ${ }^{1,2}$, Dorthe Raben 3 , Stine F Jakobsen ${ }^{3}$, Fiona Burns ${ }^{2,4}$, Andrew Copas ${ }^{2}$, Alison E Brown ${ }^{1}$, Valerie C Delpech ${ }^{1}$, on behalf of OptTEST by HIV in Europe $e^{1,2,3,4}$

1. National Infection Service, Public Health England, 61 Colindale Avenue, London, United Kingdom

2. Institute for Global Health, University College London, Mortimer Market Centre, Capper Street, London, United Kingdom

3. Center of Excellence for Health, Immunity and Infections, Rigshospitalet, University of Copenhagen, Blegdamsvej 9, Copenhagen, Denmark

4. Royal Free London NHS Foundation Trust, Pond Street, London, United Kingdom

Correspondence: Sara Croxford (sara.croxford@phe.gov.uk)

Citation style for this article:

Croxford Sara, Raben Dorthe, Jakobsen Stine F, Burns Fiona, Copas Andrew, Brown Alison E, Delpech Valerie C, on behalf of OptTEST by HIV in Europe. Defining linkage to care following human immunodeficiency virus (HIV) diagnosis for public health monitoring in Europe. Euro Surveill. 2018;23(48):pii=1700858. https:// doi.org/10.2807/1560-7917.ES.2018.23.48.1700858

Article submitted on 22 Dec 2017 / accepted on 13 May 2018 / published on 29 Nov 2018

Prompt linkage to human immunodeficiency virus (HIV) care after diagnosis is crucial to ensure optimal patient outcomes. However, few countries monitor this important public health marker and different definitions have been applied, making country and study comparisons difficult. This article presents an expert-agreed, standard definition of linkage to care for a pragmatic approach to public health monitoring, appropriate to the European context. Here, linkage to care is defined as patient entry into specialist HIV care after diagnosis, measured as the time between the HIV diagnosis date and one of the following markers: either the first clinic attendance date, first $\mathrm{CD}_{4}{ }^{+}$cell count or viral load date, or HIV treatment start date, depending on data availability; Linkage is considered prompt if within 3 months of diagnosis. Application of this definition by researchers and public health professionals when reporting surveillance or research data relating to linkage to care after HIV diagnosis will enable reliable comparisons across countries, better assessment of the success of health services programmes aimed at improving peoples access to HIV treatment and care and the identification of barriers limiting access to HIV care across Europe.

\section{Background}

Optimal health outcomes for people with human immunodeficiency virus (HIV) are dependent on diagnosis early in infection, prompt linkage to HIV services after diagnosis, engagement and retention in care and initiation of and adherence to antiretroviral therapy (ART) $[1,2]$. Late diagnosis of HIV and delays in accessing care and treatment continue to be associated with high mortality rates among people with HIV, particularly in the first year of diagnosis [3].
Public health monitoring of the HIV patient pathway, or continuum of care, is essential to ensure missed opportunities are identified and gaps closed [4]. Research from Europe has focussed on the Joint United Nations Programme on HIV/AIDS (UNAIDS) 90-90-90 targets [5], producing comparable measures for the proportion of people infected with HIV that are diagnosed, on treatment and virally suppressed [6].

However, there are little European data available on linkage to HIV care after diagnosis. A 2014 survey of European countries found there to be little agreement across countries in how to define linkage to care [4]. This lack of consensus is also reflected in the literature from Europe, in which a variety of definitions of linkage to care have been applied, making it difficult to compare across studies [7]. The use of a standard definition would be helpful to more effectively monitor entry into HIV care and facilitate comparisons across countries and populations, target interventions and identify people most at risk of delaying access to care.

In May 2015, the World Health Organization (WHO) released strategic information guidelines to consolidate key indicators to monitor the public health response to HIV [8]. These guidelines recommended that linkage to care be defined as the duration of time starting with HIV diagnosis and ending with enrolment in HIV care or treatment. However, this definition was ambiguous as to what data could be used to populate the linkage indicator and made no recommendations in what time period to use to define prompt linkage.

\section{Proposed definition}

As part of the Second Health Programme, the European Commission co-funded the Optimising testing and 
Number of new human immunodeficiency virus (HIV) diagnoses made in 2014 and data availability to monitor subsequent HIV care at a national level, European Union and European Economic Area countries, September 2016 (n = 24 countries)

\begin{tabular}{|c|c|c|c|c|c|}
\hline Country & New HIV diagnoses in $2014^{a}$ & Care attendance & $\mathrm{CD}_{4}{ }^{+}$count & Viral load & ART initiation \\
\hline Belgium & 1,050 & $\mathrm{NC}$ & $\mathrm{NC}$ & $\mathrm{NC}$ & NC \\
\hline Croatia & 92 & $\mathrm{~V}$ & $\mathrm{v}$ & $\mathrm{v}$ & $\mathrm{v}$ \\
\hline Cyprus & 56 & $\mathrm{x}$ & $\checkmark$ & $\checkmark$ & $\checkmark$ \\
\hline Czech Republic & 232 & $\mathrm{~V}$ & $\mathrm{v}$ & $\mathrm{v}$ & $x$ \\
\hline Denmark & 256 & $\mathrm{x}$ & $\mathrm{V}^{\mathrm{b}}$ & $x$ & $\mathrm{x}$ \\
\hline Estonia & 291 & $x$ & $\checkmark$ & $x$ & $x$ \\
\hline Finland & 181 & $x$ & $\sqrt{ }^{\mathrm{b}}$ & $x$ & $x$ \\
\hline France & 5,653 & $\mathrm{x}$ & $\checkmark$ & $\checkmark$ & $x$ \\
\hline Germany & 3,500 & $\mathrm{x}$ & $\mathrm{v}$ & $\checkmark$ & $x$ \\
\hline Greece & 761 & $x$ & $\checkmark$ & $\mathrm{x}$ & $x$ \\
\hline Ireland ${ }^{c}$ & 363 & $x$ & $V^{b}$ & $\mathrm{~V}^{\mathrm{b}}$ & $x$ \\
\hline Italy & 3,850 & $x$ & $\checkmark$ & $\checkmark$ & $x$ \\
\hline Latvia & 347 & $\mathrm{x}$ & $\checkmark$ & $x$ & $x$ \\
\hline Lithuania & 141 & $x$ & $V^{b}$ & $\mathrm{~V}^{\mathrm{b}}$ & $\mathrm{V}^{\mathrm{b}}$ \\
\hline Luxembourg & 74 & $\checkmark$ & $\checkmark$ & $\checkmark$ & $\checkmark$ \\
\hline Malta & 40 & $\checkmark$ & $\checkmark$ & $\checkmark$ & $x$ \\
\hline The Netherlands & 881 & $\mathrm{~V}$ & $\mathrm{v}$ & $\mathrm{v}$ & $\mathrm{v}$ \\
\hline Norway & 267 & $x$ & $\mathrm{x}$ & $x$ & $x$ \\
\hline Poland & 1,133 & $x$ & $x$ & $x$ & $x$ \\
\hline Portugal & 1,109 & $x$ & $V^{b}$ & $V^{b}$ & $x$ \\
\hline Romania & 825 & $\mathrm{~V}$ & 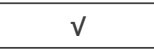 & $\mathrm{V}$ & $\mathrm{v}$ \\
\hline Slovenia & 49 & $x$ & $\checkmark$ & $x$ & $x$ \\
\hline Spain & 4,140 & $x$ & $\checkmark$ & $x$ & $x$ \\
\hline UK & 6,157 & $\mathrm{~V}$ & $\checkmark$ & $\checkmark$ & $\checkmark$ \\
\hline
\end{tabular}

NC: Not complete (country responded to other parts of survey but did not complete data table); v: data available; $\mathrm{x}$ : no data available; UK: United Kingdom.

a Data from ECDC [26].

${ }^{b}$ No date information collected.

c Data on ART initiation and CD4+ and viral load dates collected from 2015 onwards.

linkage to care for HIV across Europe (OptTEST) project, with an aim to optimise testing and linkage to care for HIV in Europe through a series of work packages [9]. In September 2015, OptTEST hosted a workshop on standardising the linkage to care indicator at a wider expert meeting on the continuum of care hosted by the European Centre for Disease Prevention and Control (ECDC) [10]. The meeting was attended by 48 experts from 20 countries, including HIV surveillance leads from the European HIV Surveillance Network and representatives from European HIV patient cohorts. A full list of attendees can be found in the Supplement. Workshop discussions resulted in experts advocating for a pragmatic approach to monitoring linkage to care in Europe.

The WHO definition was endorsed and further operationalised, with linkage to care defined as patient entry into specialist HIV care after diagnosis, measured as the time between the HIV diagnosis date and either the first clinic attendance date, first $\mathrm{CD}_{4}{ }^{+}$count or viral load date, or HIV treatment start date, depending on data availability. While, first clinic date after diagnosis was considered the gold standard marker for linkage to care, experts agreed that for many countries, routine baseline laboratory data, such as first $\mathrm{CD}_{4}{ }^{+}$and viral load date, would be practical proxies for care entry. The WHO currently recommends $\mathrm{CD}_{4}{ }^{+}$and viral load testing to be carried out at baseline for all newly diagnosed persons at the first clinic visit [11]. Treatment initiation was included as a marker for care to acknowledge the change in guidelines that state people diagnosed with HIV should start ART as soon as possible, regardless of $\mathrm{CD}_{4}^{+}$count, for optimal outcomes [12]. In addition, some countries in Europe with limited surveillance may not be able to collect bio-marker or clinic data but may have information on ART initiation linked to external funding for national treatment programmes. For countries with available data on multiple care indicators, first clinic date was preferred, followed by $\mathrm{CD}_{4}{ }^{+}$count date, viral load measurement date and finally, treatment start date. 
There was also discussion at the workshop around the accuracy of care indicator dates. It was recognised that the dates captured by surveillance systems across Europe vary and this may influence linkage calculations. Where possible, care attendance date should be captured as the date of first visit to the HIV specialist care provider, regardless of visit purpose. $\mathrm{CD}_{4}^{+}$and viral load dates should be the date that the patient blood sample was taken for testing at the specialist clinic. ART initiation date should reflect the clinic visit at which patients were first prescribed ART.

Prompt linkage to care was defined by experts as linkage to care within 3 months of diagnosis, in-line with guidance from the United States (US) Centers for Disease Prevention and Control (CDC) [13]. The decision to recommend a 3 month cut-off in Europe was based on research from the US showing that initiation of care within 3 months of HIV diagnosis is significantly associated with faster time to viral suppression [2]. Furthermore, data from the United Kingdom show that $>95 \%$ of people diagnosed with HIV enter care within 3 months of diagnosis [14] and a 3 month cut-off has been used widely in the literature from Europe [7].

\section{Developmental approach}

The expert-agreed definition of linkage to care utilises a number of markers to signify entry into HIV care, acknowledging that there are disparate models of care across Europe and data used to calculate linkage are captured to varying extents by different countries $[15,16]$.

In 2016, a survey was developed as part of the OptTEST project, in collaboration with international experts from the ECDC, the WHO Regional Office for Europe, the HIV/AIDS Civil Society Forum, the EURO HIV EDAT (Operational knowledge to improve HIV early diagnosis and treatment among vulnerable groups in Europe) project, the European AIDS Treatment Group, and national experts from Public Health England and AIDS Fondet. The 30 national surveillance contact points for HIV in the European Union/European Economic Area (EU/ EEA) were identified and invited to take part. The main objectives were to assess variables collected at the national level that could be used to monitor linkage to care, identify data caveats and receive feedback on the proposed definition. National data on diagnoses of HIV made between 2010 and 2014 were requested, alongside the proportion of those diagnoses that had entry marker data available ever after diagnosis and within 3 months of diagnoses (e.g. the proportion of people with a viral load available).

Responses were received from 24 (80\%) of 30 EU/EEA national contact points (Table). Nineteen countries provided data on the number of new HIV diagnoses with at least one marker indicating subsequent care. Four countries commented on data availability but did not, or were not able, to provide data. Taking into account both the submitted data and narrative responses, linkage to care should be able to be calculated using the time difference between diagnosis date and (i) care attendance date in seven countries (ii) $\mathrm{CD}_{4}^{+}$date in 16 countries, (iii) viral load date in 11 countries and (iv) treatment initiation in six countries. Five countries collected markers of linkage to care but not the marker date, allowing calculation of the proportion ever linked to care but not the timeliness of linkage. The number of new HIV diagnoses in 2014 has been included in Table to show the relative size of the epidemics in each country, in the absence of prevalence data.

The majority of countries not able to provide attendance or treatment data, cited problems with either the variable not being collected at all (attendance date: $\mathrm{n}=9$; treatment start: $\mathrm{n}=10$ ) and/or data not being reported centrally (attendance date: $n=14$; treatment start: $n=12$ ). The most common reasons for difficulty in providing $\mathrm{CD}_{4}^{+}$information was incomplete reporting to national surveillance by clinicians $(n=8)$ and when data were provided, significant reporting delay $(n=3)$. Viral load was more difficult to report than CD4+ because of a lack of centralised data collection mechanisms $(n=8)$. There were a few countries that reported issues collecting longitudinal patient data after diagnosis as care data were either housed in a separate clinical cohort database rather than collected as part of national surveillance $(n=3)$ or there was no legal framework for collection $(n=6)$.

\section{Discussion and conclusions}

The expert-agreed definition of linkage to care presented here provides a pragmatic approach to the public health monitoring of this key HIV indicator that is appropriate to the European context. Linkage to care should be defined as patient entry into specialist HIV care after diagnosis, more specifically, the time between the HIV diagnosis date and either the first clinic attendance date, first $\mathrm{CD}_{4}{ }^{+}$count, viral load date, or HIV treatment start date, with prompt linkage measured within 3 months. The application of this definition by researchers and public health professionals alike when reporting surveillance or research data relating to linkage to care after HIV diagnosis will enable reliable comparisons across countries, studies, population groups and allow monitoring of changes in linkage to care over time. The identification of gaps in linkage to care will allow for better insight into the barriers that may currently be limiting access to HIV medical care across Europe.

In order to apply this definition, national public health agencies and institutions should ensure adequate capture of clinical data on HIV diagnosis and entry in to care, such as $\mathrm{CD}_{4}{ }^{+}$cell count. Of the 24 countries who responded to the survey, 16 could adopt this definition given their current national HIV surveillance system. However, eight reported poor completion rates of care marker data and 14 reported issues with collecting data centrally, which may require additional resources and time. An analysis of European HIV surveillance data 
revealed that even $\mathrm{CD}_{4}^{+}$data, which was considered the most appropriate indicator of linkage to care by EU/ EEA countries, was only complete for ca $46 \%$ of new HIV diagnoses in the WHO European Region reported to the ECDC/WHO [17].

To determine the extent to which this expert definition is already being used in Europe, a systematic review of the literature was carried out in February 2017 [18]. Results indicate that 24 studies used the agreed definition to calculate linkage to care. Of the 22 that presented estimates, only 14 used a 3 month cut-off for prompt linkage. However, this definition and cut-off has also been applied to US, Canadian and Australian data [19]. To explore barriers to national monitoring of linkage to care using this definition and better understand the context within which linkage occurs, OptTEST organised meetings in collaboration with local partners in Greece, Poland and Portugal, bringing together key stakeholders from government, public health, community organisations and HIV care centres. These meetings highlighted the variability in the way HIV care and HIV surveillance systems are structured in each country [20-22], which is reflected in the flexibility in care markers used in the expert-agreed linkage to care definition.

The ability to calculate linkage to care also depends on robust reporting of date of HIV diagnosis. Although in discussion at the expert workshop, the importance of capturing data on a patient's first reactive test was highlighted, it was recognised that the most practical date of diagnosis is the date the laboratory sample was taken for confirmatory HIV testing.

It is important to acknowledge that the agreed definition presented here is most appropriate for monitoring linkage to care at a national or clinic level and potentially less applicable to local community testing facilities. Also, co-funded by the European Commission, the Euro HIV EDAT project defined linkage to care as: "entry into health care or follow-up by an HIV specialist or in an HIV-unit after a reactive or confirmatory HIV test at a community testing facility.' [23] Researchers found that though this was the most practical definition, community testing organisations across Europe face problems obtaining reliable information on whether a patient was successfully linked to care because of confidentiality and data protection issues. Often reporting of linkage is informal and limited date information is collected [23,24].

In conclusion, adoption of the expert-agreed definitions of linkage to care and prompt linkage presented here is needed to ensure consistent monitoring of the equitable access to HIV care and treatment. Application of a standard definition will help to better to understand time trends and to identify and compare populations most at risk of not attending for HIV care after diagnosis across Europe. The ECDC and the WHO Regional Office for Europe should continue to work with European countries to improve reporting of linkage to care data for public health monitoring. In addition, collaboration between public health bodies and national HIV clinical cohorts should be strengthened to address gaps in care data availability [25].

\section{Acknowledgements}

This paper was co-funded by the European Commission and Public Health England as part of OptTEST by HIV in Europe (Grant \#: 20131102). We would like to thank the ECDC, the European HIV Surveillance Network and all other attendees of the continuum of care meeting for their contributions toward developing the definition. We would also like to acknowledge our OptTEST and Euro HIV EDAT project partners for their contributions and input in putting together this manuscript, as well as the OptTEST project steering committee: Jorrit Kabel (AIDS Fonds), Anne-Isabelle Von Lingen (EATG), Ann Sullivan (St Stephen's AIDS Trust), Yazdan Yazdanpanah (INSERM), Julian Hows (GNP+), Julia Del Amo (ISC III), Kristi Rüütel (NIHD - Estonia) and Jens Lundgren (CHIP). Finally, we would like to thank the ECDC, the WHO Regional Office for Europe and the ECDC/WHO national HIV surveillance focal points for their involvement in the OptTEST survey.

\section{Conflict of interest}

None declared.

\section{Authors' contributions}

All authors were involved in the development of the OptTEST survey on linkage to care and contributed important intellectual content to this manuscript. All authors commented on the manuscript and approved the final draft. SC was scientific lead for work package 4 of the OptTEST project and was responsible for creating the survey and carrying out data analysis and interpretation. SC also drafted the manuscript, incorporated author comments, and was responsible for the final draft to be published. DR, SFJ, FB, AC, AB and VD were involved in survey data analysis interpretation and contributed to the discussion and conclusions. VD also contributed to the study conception and acted as the principal investigator for work package 4 of OptTEST.

\section{References}

1. Ulett KB, Willig J H, Lin $\mathrm{H}-\mathrm{Y}$, Routman IS, Abroms S, Allison J, et al. The therapeutic implications of timely linkage and early retention in HIV care. AIDS Patient Care STDS. 2009;23(1):41-9. https://doi.org/10.1089/apc.2008.0132 PMID: 19055408

2. Robertson M, Laraque F, Mavronicolas H, Braunstein S, Torian L. Linkage and retention in care and the time to HIV viral suppression and viral rebound - New York City. AIDS Care. 2015;27(2):260-7. https://doi.org/10.1080/09540121.2014.959 463 PMID: 25244545

3. Croxford S, Kitching A, Desai S, Kall M, Edelstein M, Skingsley A, et al. Mortality and causes of death in people diagnosed with HIV in the era of highly active antiretroviral therapy compared with the general population: an analysis of a national observational cohort. Lancet Public Health. 2017;2(1):e35-46. PMID: 29249478

4. Drew RS, Rice B, Rüütel K, Delpech V, Attawell KA, Hales DK, et al. HIV continuum of care in Europe and Central Asia. HIV Med. 2017;18(7):490-9. https://doi.org/10.1111/hiv.12480 PMID: 28117527

5. Joint United Nations Programme on HIV/AIDS (UNAIDS). 90-9090 an ambitious target to help end the AIDS epidemic. Geneva: UNAIDS; 2014. Available from: http://www.unaids.org/sites/ default/files/media_asset/90-90-90_en.pdf 
6. European Centre for Disease Prevention and Control (ECDC). Continuum of HIV care - technical report. Stockholm: ECDC; 2017. Available from: https://ecdc.europa.eu/sites/portal/ files/documents/Continuum-of-HIV-care-2017.pdf

7. Croxford S, Raben D, Burns F, Delpech V. OptTEST by HIV in Europe. PE21/15 Linkage to care following HIV diagnosis in Europe: a review of the literature. European AIDS Clinical Society Conference. Barcelona, Spain, October 2015.

8. World Health Organization (WHO). Consolidated strategic information guidelines for HIV in the health sector. Geneva: WHO; 2015. Available from: http://www.who.int/hiv/pub/ guidelines/strategic-information-guidelines/en/

9. Optimising testing and linkage to care for HIV across Europe (OptTEST). About OptTEST. Available from: http://www.opttest. $\mathrm{eu} /$

10. European Centre for Disease Prevention and Control (ECDC). Meeting report: Optimising analysis of the HIV continuum of care in Europe. Stockholm: ECDC; 2015 . Available from: https://ecdc.europa.eu/en/news-events/ecdc-expert-meetingoptimising-analysis-hiv-continuum-care-europe

11. World Health Organization (WHO). Consolidated guidelines on person-centred HIV patient monitoring and case surveillance. Geneva: WHO; 2017. Available from: http://www.who.int/hiv/ pub/guidelines/person-centred-hiv-monitoring-guidelines/en/

12. World Health Organization (WHO). Consolidated guidelines on the use of antiretroviral drugs for treating and preventing HIV infection. Geneva: WHO; 2016. Available from: http://www. who.int/hiv/pub/arv/arv-2016/en/

13. Centers for Disease Control and Prevention (CDC). Understanding the HIV Care Continuum. Atlanta, Georgia: CDC; 2014. Available from: https://www.cdc.gov/hiv/pdf/ DHAP_Continuum.pdf

14. Delpech V, Brown AE, Croxford S, Chau C, Polavarapu V, Cooper N, et al. Quality of HIV care in the United Kingdom: key indicators for the first 12 months from HIV diagnosis. HIV Med. 2013;14(Suppl 3):19-24. https://doi.org/10.1111/hiv.12070 PMID: 24033898

15. European Centre for the Epidemiological Monitoring of HIV/ AIDS WHO and UNAIDS Collaborating Centre on HIV/AIDS (EuroHIV). Report on the EuroHIV 2006 survey on HIV and AIDS surveillance in the WHO European Region. Saint-Maurice: Institut de veille sanitaire; 2007. Available from: http://opac. invs.sante.fr/doc_num.php?explnum_id=3591

16. Organisation for Economic Co-operation and Development $(\mathrm{OECD} / \mathrm{EU})$. Health at a Glance: Europe 2016 - state of health in the EU cycle. Paris: OECD Publishing; 2016. Available from: http://www.oecd.org/health/health-at-a-glanceeurope-23056088.htm

17. Croxford S, Burns F, Copas A, Pharris A, Rinder Stengaard A, Delpech VOptTEST by HIV in Europe. Factors associated with delayed linkage to care following HIV diagnosis in the WHO European Region. HIV Med. 2018;19(Suppl 1):40-6. https://doi. org/10.1111/hiv.12585 PMID: 29488702

18. Croxford S, Yin Z, Burns F, Copas A, Town K, Desai S, et al. OptTEST project. Linkage to HIV care following diagnosis in the WHO European Region: A systematic review and meta-analysis, 2006-2017. PLoS One. 2018;13(2):e0192403. https://doi. org/10.1371/journal.pone.0192403 PMID: 29451875

19. Hall HI, Halverson J, Wilson DP, Suligoi B, Diez M, Le Vu S, et al. Late diagnosis and entry to care after diagnosis of human immunodeficiency virus infection: a country comparison. PLoS One. 2013;8(11):e77763. https://doi.org/10.1371/journal. pone.0077763 PMID: 24223724

20. Optimising testing and linkage to care for HIV across Europe (OptTEST). Meeting Report: National stakeholder meeting on implementation of HIV testing in primary care \& linkage to care in Portugal. Portugal: OptTEST; 2017. Available from: http:// www.opttest.eu/Portals/o/Meeting_report_Portugal_final.pdf

21. Optimising testing and linkage to care for HIV across Europe (OptTEST). Linkage to and retention in care: Experiences from Greece and challenges for the future - OptTEST meeting report. Greece; OptTEST; 2017. Available from: http://www.opttest. eu/Portals/o/Publications/OptTEST_meeting\%2oreport_ greece_26SEP2016_final.pdf

22. Kowalska JD, Grzeszczuk A, Pyziak-Kowalska K, MarzecBogusławska A, Rosińska M, Ankiersztejn-Bartczak M, et al. Shaping the HIV epidemic in Poland - proceedings from the first Polish workshop on cascade of care in HIV. HIV \& AIDS Review. International Journal of HIV-Related Problems.2017;16(2):130-3.

23. Operational knowledge to improve HIV early diagnosis and treatment among vulnerable groups in Europe (EURO HIV EDAT). Optimal linkage to care among MSM: a practical guide for CBVCT's and points of care. Copenhagen: EURO HIV EDAT; 2017. Available from: https://eurohivedat.eu/arxius/
ehe_docsmenu_docsmenu_doc_128-20131101_Do5_0o OTH_1_EN_PS.p.df

24. Operational knowledge to improve HIV early diagnosis and treatment among vulnerable groups in Europe (EURO HIV EDAT). WP 6- Description and improvement of different approaches of linkage to care for HIV among MSM in Europe. Copenhagen: EURO HIV EDAT; 2017. Available from: https:// eurohivedat.eu/arxius/ehe_docsmenu_docsmenu_doc_127EURO HIV EDAT_WP 6 Data_Report FINAL.pdf

25. Gourlay A, Noori T, Pharris A, Axelsson M, Costagliola D, Cowan S, et al. European HIV Continuum of Care Working Group. The Human Immunodeficiency Virus continuum of care in European Union countries in 2013: data and challenges. Clin Infect Dis. 2017;64(12):1644-56. https://doi.org/10.1093/cid/ cix212 PMID: 28369283

26. European Centre for Disease Prevention and Control (ECDC), World Health Organisation (WHO) Regional Office for Europe. HIV/AIDS surveillance in Europe 2015. Stockholm: ECDC; 2016. Available from: http://www.euro.who.int/ data/assets/pdf file/0019/324370/HIV-AIDS-surveillance-Europe-2015.pdf

\section{License and copyright}

This is an open-access article distributed under the terms of the Creative Commons Attribution (CC BY 4.0) Licence. You may share and adapt the material, but must give appropriate credit to the source, provide a link to the licence, and indicate if changes were made.

This article is copyright of the authors or their affiliated institutions, 2018. 